\title{
Pressions anthropiques sur la forêt de Besalampy : richesse faunique, caractère socio-économique et accords pour la création d'une nouvelle aire protégée
}

Riana V. Ramanantsalamal, ", Tantely H. Razafiarisolol, ", Andriamihaja Rasolohery', "I

\section{RÉSUMÉ}

Des inventaires biologiques et des enquêtes socio-économiques ont été effectués dans un site potentiel pour la protection de la biodiversité, subissant une forte pression anthropique, à Besalampy, ouest de Madagascar, afin de créer un projet de conservation durable. Quatre espèces de lémuriens (Eulemur rufifrons, Hapalemur occidentalis, Lepilemur ruficaudatus, Propithecus deckenii), 31 espèces d'oiseaux avec des espèces appartenant aux familles (Vangidae : Leptopterus viridis) et sousfamilles endémiques (Couinae : Coua coquereli et C. cristata) malgaches; ; et la tortue endémique en danger critique d'extinction Erymnochelys madagascariensis, ont été échantillonnées. La création d'une nouvelle aire protégée pourrait profiter à la fois à la protection de la biodiversité et à l'amélioration du bien-être des villageois vivant à proximité de cette zone de Besalampy.

\section{ABSTRACT}

Based on the Réseau de la Biodiversité de Madagascar (ReBioma), the forest of Besalampy, western Madagascar, is a potential site to protect the biodiversity, although subject to high anthropogenic pressure. Hence, biological and socio-economic surveys have been made in order to create a sustainable conservation project. Direct observations, and song and call listening technics were used to list species of lemurs, reptiles, amphibians and birds along predefined transects. Some birds have been captured using nets. Thus, four species of lemur (Eulemur rufifrons, Hapalemur occidentalis, Lepilemur ruficaudatus, Propithecus deckenii), 31 species of bird including those belonging to Malagasy endemic family (Vangidae: Leptopterus viridis) and subfamily (Couinae: Coua coquereli and C. cristata), and the endemic critically endangered big-headed turtle Erymnochelys madagascariensis were sampled. The habitat loss resulting from anthropogenic activities and hunting threatened the biodiversity in Besalampy. The poor living condition forced the local community to use the forest resources in an irrational manner, and
Correspondence:

Riana V. Ramanantsalama

Mention Zoologie et Biodiversité Animale, Université

d'Antananarivo, BP 906, Antananarivo 101, Madagascar

Email: rianavaleryb@gmail.com

the creation of a new protected area should provide a conciliation between biodiversity and local people.

\section{INTRODUCTION}

À Madagascar, les activités humaines de ces dernières décennies ont conduit à la disparition de $44 \%$ de la couverture forestière entre 1953 et 2014 en se basant sur les images satellitaires (Vieilledent et al. 2018) ; les forêts caducifoliées ont été les plus touchées. Se trouvant dans la partie ouest de Madagascar, le district de Besalampy présente une forêt sèche caducifoliée abritant des espèces endémiques emblématiques malgaches, notamment les lémuriens (Ralison 2007), la chauve-souris à ventouses de Schliemann Myzopoda schliemanni de la famille endémique malgache des Myzopodidae, Microgale brevicaudata de la famille endémique des Tenrecidae (Rajemison et Goodman 2007, Soarimalala 2011) ou le pygargue de Madagascar en danger critique d'extinction CR Haliaeetus vociferoides de la famille des Accipitridae (Razafimanjato et al. 2013). Les zones humides de Besalampy abritent la râle d'Olivier en danger d'extinction CE Zapornia olivieri (Rallidae) (Rabenandrasana et al. 2009) et une tortue d'eau douce, la réré, en danger critique d'extinction CR Erymnochelys madagascariensis (Podocnemididae) (Rabenandrasana 2007). Ces zones humides s'étendent jusqu'au littoral, en passant par les estuaires, et sont connues par la présence des lémuriens dans les mangroves (Gardner 2016, Donati et al. 2019). Toutefois, les forêts de Besalampy ont subi un important défrichement durant ces dernières décennies (Vieilledent et al. 2018).

Le district de Besalampy est difficile d'accès par voie routière et sa biodiversité est encore mal connue même par les biologistes (Goodman et Raselimanana 2008). Les inventaires biologiques dans ce district étaient surtout concentrés dans sa partie nord, notamment Andranomahitsy à $\mathrm{E} 044^{\circ} 29^{\prime}$ et $\mathrm{S} 16^{\circ} 31^{\prime}$, avec des recensements de micromammifères et de lémuriens (Rajemison et

\footnotetext{
I Mention Zoologie et Biodiversité Animale, Université d'Antananarivo, BP 906, Antananarivo 101, Madagascar

॥ Association Vakoka, Antananarivo 101, Madagascar

Citation Ramanantsalama, R. V., Razafiarisolo, T. H., Rasolohery, A. 2021. Pressions anthropiques sur la forêt de Besalampy: richesse faunique, caractère socioéconomique et accords pour la création d'une nouvelle aire protégée. Madagascar Conservation \& Development 16, 1: 43-47. http://dx.doi.org/10.4314/mcd.v16i1.3
} 


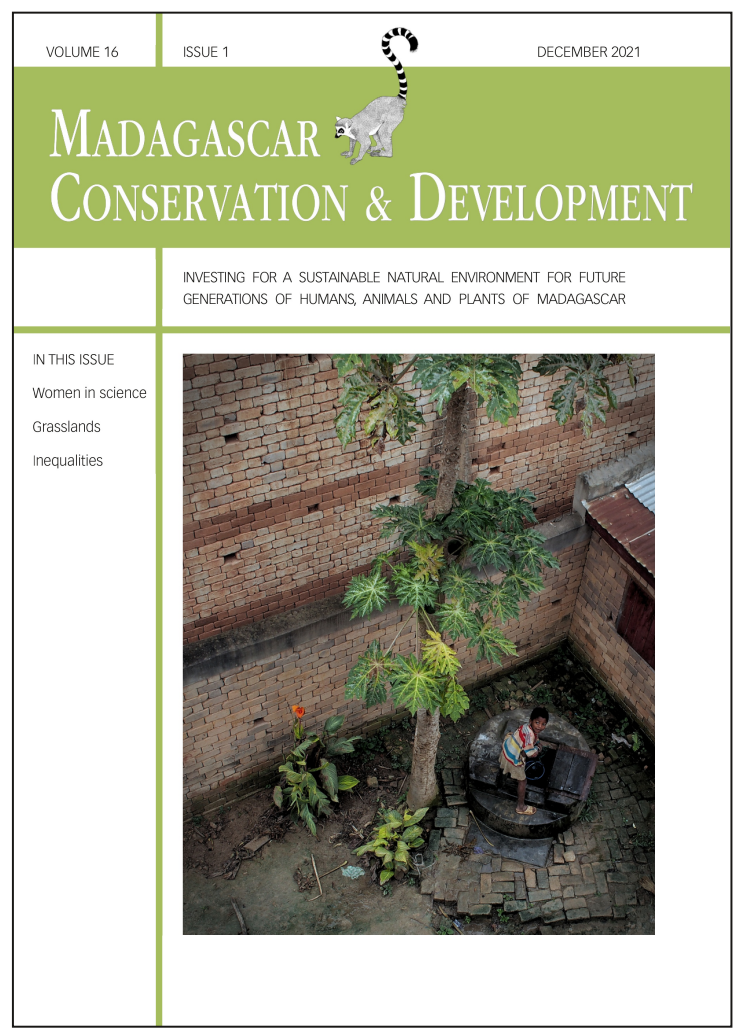

Madagascar Conservation \& Development is the journal of Indian Ocean e-Ink. It is produced under the responsibility of this institution. The views expressed in contributions to MCD are solely those of the authors and not those of the journal editors or the publisher.

All the Issues and articles are freely available at https://www.journalmcd.com

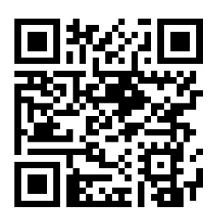

Contact Journal MCD

info@journalmcd.net for general inquiries regarding MCD funding@journalmcd.net to support the journal

Madagascar Conservation \& Development Institute and Museum of Anthropology

University of Zurich

Winterthurerstrasse 190

CH-8057 Zurich

Switzerland

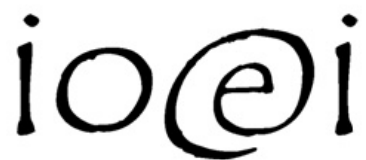

Indian Ocean e-Ink

Promoting African Publishing and Education

www.ioeink.com

Missouri Botanical Garden (MBG)

Madagascar Research and Conservation Program

敨 Missouri Botanical Garden BP3391

Antananarivo, 101, Madagascar 
Goodman 2007, Ralison 2007, Soarimalala 2011) ; et sa partie sud notamment les Réserves Spéciales de Bemarivo et Maningoza res-

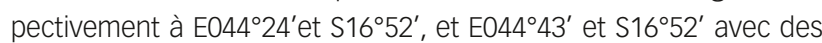
études sur la flore, les insectes, les oiseaux et les primates (Fishpool et Evans 2001, Schatz et al. 2001, Rasamison et al. 2005, Randrianarisoa et al. 2000, Forthman et al. 2016). Par conséquent, la présente étude se focalise sur des inventaires biologiques et des enquêtes socio-économiques dans un site potentiel pour la protection de la biodiversité selon le Réseau de la Biodiversité de Madagascar (ReBioma), dans le district de Besalampy (en dehors des Réserves Spéciales de Bemarivo et de Maningoza), qui selon nos connaissances n'a pas encore été visité par les chercheurs en biologie et écologie. L'objectif est de présenter les espèces de vertébrés et le mode de vie des gens de la région ainsi que leurs activités en relation avec la nature. Ce travail consiste à mettre en évidence les pressions sur la faune et la flore sauvage de Besalampy afin d'envisager des projets de développement et de conservation durables.

\section{MÉTHODOLOGIE}

INVENTAIRES FAUNIQUES. Les inventaires ont été réalisés du

21 juillet au 9 août 2016 dans la forêt de Besalampy (entre les longitudes $\mathrm{E} 44^{\circ} 21^{\prime}$ et $\mathrm{E} 044^{\circ} 28^{\prime}$ et les latitudes $\mathrm{S} 16^{\circ} 43^{\prime}$ et $\mathrm{S} 16^{\circ}$ $49^{\prime}$ ), plus précisément dans les stations d'Andranomanenty (E044 $28^{\prime}$ et $\left.\mathrm{S} 16^{\circ} 44^{\prime}\right)$, Andranovorombahoaka (E044 $26^{\prime}$ et $\mathrm{S} 16^{\circ} 47^{\prime}$ ), An-

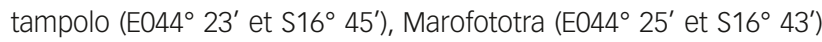
et Marotondro ( $\left(\mathrm{E} 044^{\circ} 21^{\prime}\right.$ et $\left.\mathrm{S} 16^{\circ} 47^{\prime}\right)$. Le protocole d'échantillonnage des espèces d'oiseaux a été adapté à partir des méthodes standards utilisées sur des lignes d'observation directe et d'écoute (Bibby et al. 1992). Les caractères morphologiques et les chants des oiseaux ont été identifiés en se basant sur le guide de Hawkins et al. (2015). Dans la présente étude, deux lignes d'échantillonnage d'un kilomètre de longueur ont été choisies : (i) une ligne traversant la forêt intacte, et (ii) une ligne traversant les formations perturbées et anthropiques. Les espèces d'oiseaux ont été inventoriées d'une manière continue le long de ces lignes entre 0500 et 0700 durant trois jours dans chaque station en se déplaçant à une vitesse constante d'environ $2 \mathrm{~km} / \mathrm{h}$. Trois filets japonais de $12 \mathrm{~m} \times 2 \mathrm{~m}$ ont été déployés près du campement dans chaque station pour capturer des oiseaux de sous-bois. Après que ces derniers ont été identifiés, ils sont ensuite relâchés entre 0500 et 0700 au voisinage des lignes d'échantillonnage

Pour les carnivores endémiques malgaches (Eupleridae), des enquêtes auprès de la population locale appuyées par des observations directes et des prospections d'éventuelles traces de présence, notamment les traces de pattes et les fèces, ont été réalisées pour recenser les présences. Toutefois, les informations précises destinées à l'identification des traces de pattes et des fèces des Eupleridae sont limitées aux recherches faites par Albignac (1970) et Hawkins et Racey (2008) sur des Cryptoprocta ferox ; la reconnaissance des autres Eupleridae d'une manière indirecte s'avéraient ainsi difficile. Des observations directes suivant les mêmes lignes d'échantillonnage que les oiseaux ont été effectuées pour inventorier les espèces de lémurien.

La présence des espèces d'amphibiens et de reptiles dans le site d'étude a été déterminée par la technique d'observation directe et la fouille systématique des microhabitats suivant deux lignes d'échantillonnage de $250 \mathrm{~m}$ pour les amphibiens et $1 \mathrm{~km}$ pour les reptiles. Pour chacun des deux groupes, une ligne d'échantillonnage traversait la forêt intacte et une autre ligne pas- sait par les formations perturbées et anthropiques. Les informations sur les espèces de poissons ont été recueillies auprès des pêcheurs en se basant sur les critères d'identification de King (1997) ainsi que Rham et Nourissat (2004).

ENQUÊTES SOCIO-ÉCONOMIQUES. La méthode d'enquête choisie a été adoptée à partir des techniques d'entretien individuel effectuées par Marcus (2001) qui a étudié la perception de la population locale vis-à-vis de la création des aires protégées à Masoala (partie nord-est de Madagascar) et Ranomafana (partie est de Madagascar). Dans le présent travail, après un entretien avec le président du «fonkontany » couvrant les stations d'Andranomanenty, d'Andranovorombahoaka, d'Antampolo et de Marofototra, les personnes adultes (> 18 ans) résidant dans ces stations ont été invitées à une réunion d'explication de l'objectif de l'investigation. Des volontaires ont été sélectionnés au hasard à partir de la fiche de présence à la réunion pour être enquétés ; les questionnaires d'enquête portaient sur les sources et le montant du revenu mensuel, le montant et la nature des dépenses familiales mensuels ainsi que la perception sur l'utilité de la forêt et de la biodiversité.

\section{RÉSULTATS}

FAUNE DE LA FORÊT DE BESALAMPY. Des groupes de Propithecus deckenii ont été observés dans toutes les stations de collecte de données (Tableau 1) ; les Lepilemur ruficaudatus ont été vus dans les stations d'Antampolo et de Marofototra, les Hapalemur occidentalis dans la station d'Antampolo et Eulemur rufifrons dans la station de Marotondro. Chez les Eupleridae, aucun individu n'a été rencontré ; toutefois, des traces de pas observées sur le littoral de Marofototra ont suggéré la présence de Cryptoprocta ferox.

Dans le souci de perdre les filets pour les captures des oiseaux, les filets n'ont pas été utilisés dans les stations d'Andranomanenty et d'Antampolo où les activités de banditisme et de vol sont fréquentes. Un nombre total de 31 espèces d'oiseaux réparties dans 21 familles ont été inventoriées (Tableau 1), dont des rapaces se trouvant au sommet de la chaine trophique (Buteo brachypterus, Polyboroides radiatus : Accipitridae), jusqu'aux Cuculiformes et Passeriformes, particulièrement ceux qui se trouvent dans les familles (Leptopterus viridis : Vangidae) et sous-familles endémiques (Coua coquereli et C. cristata : Couinae) malgaches.

Aucune espèce d'amphibien n'a été rencontrée. Chez les reptiles, sept genres appartenant à sept familles différentes ont été inventoriés (Tableau 1) et sont tous endémiques de Madagascar. ॥ n'a pas été possible de voir des individus vivants d'Erymnochelys madagascariensis ; toutefois, la population locale affirme que l'espèce est toujours présente et des restes d'individus qui venaient d'être chassés ont pu être observés.

Les poissons inventoriés constituaient les principaux moyens de subsistances de la population locale se trouvant dans les villages de pêcheurs. Les sept espèces recensées ont été observées dans les pièges des pêcheurs au sein des zones humides ; trois espèces sont authochnones dont une seule est endémiqe de l'île (Clupeidae : Sauvagella madagascariensis), trois autres espèces sont allochtones (Tableau 1).

ENQUÊTES SOCIO-ÉCONOMIQUES. Les enquêtes ont dévoilé qu'une famille gagne de 10000 à 800000 MGA par mois, soit une moyenne de $216750 \pm 182800$ MGA par mois ( $N=20$ ), soit près de US\$ 65,7 $\pm 55,4$ par mois en 2016 . Leur principale source 
Tableau 1. Listes des espèces de vertébrés rencontrés dans la forêt de Besalampy et leur statut de conservation selon I'UICN. (CR : en danger critique d'extinction (critically endangered), EN : en danger (endangered), VU : vulnérables (vulnerable), NT : quasi-menacée (near threatened), LC : préoccupation mineur (least concern)).

\begin{tabular}{|c|c|c|c|}
\hline Groupe, Ordre & Famille & Espèce & $\begin{array}{l}\text { Statut } \\
\text { UICN }\end{array}$ \\
\hline \multirow[t]{4}{*}{ Lémuriens, Primates } & Lemuridae & Eulemur rufifrons & NT \\
\hline & & Hapalemur occidentalis & VU \\
\hline & Lepilemuridae & Lepilemur ruficaudatus & VU \\
\hline & Indridae & Propithecus deckenii & EN \\
\hline \multirow[t]{30}{*}{ Oiseaux, Aves } & Accipitridae & Accipiter spp. & - \\
\hline & & Buteo brachypterus & LC \\
\hline & & Polyboroides radiatus & LC \\
\hline & Alcedinidae & Corythornis vintsioides & LC \\
\hline & Anatidae & Thalassornis leuconotus & LC \\
\hline & Ardeidae & Egretta dimorpha & LC \\
\hline & Caprimulgidae & Caprimulgus madagascariensis & LC \\
\hline & Charadriidae & Charadrius hiaticula & LC \\
\hline & Columbidae & Oena capensis & LC \\
\hline & & Streptopelia picturata & LC \\
\hline & & Treron australis & LC \\
\hline & Corvidae & Corvus albus & LC \\
\hline & Cuculidae & Centropus toulou & LC \\
\hline & & Coua coquereli & LC \\
\hline & & Coua cristata & LC \\
\hline & Dicruridae & Dicrurus forficatus & LC \\
\hline & Meropidae & Merops superciliosus & LC \\
\hline & Monarchidae & Terpsiphone mutata & LC \\
\hline & Motacillidae & Motacilla flaviventris & LC \\
\hline & Nectarinidae & Nectarinia souimanga & LC \\
\hline & Numididae & Numida meleagris & LC \\
\hline & Phalacrocoracidae & Phalacrocorax africanus & LC \\
\hline & Ploceidae & Foudia madagascariensis & LC \\
\hline & Podicipedidae & Tachybaptus pelzelnii & LC \\
\hline & Psittacidae & Agapornis cana & LC \\
\hline & & Coracopsis vasa & LC \\
\hline & Rallidae & Dryolimnas cuvierii & LC \\
\hline & Sturnidae & Acridotheres tristis & - \\
\hline & Turdidae & copsychus albospecularis & LC \\
\hline & Vangidae & Leptopterus viridis & LC \\
\hline \multirow[t]{6}{*}{ Reptiles, Squamata } & Boidae & Acrantophis madagascariensis & $\mathrm{VU}$ \\
\hline & Colubridae & Leioheterodon madagascariensis & LC \\
\hline & Chameleonidae & Calumma sp. & - \\
\hline & Gekkonidae & Phelsuma sp. & - \\
\hline & Gerrhosauridae & Zonosaurus sp. & - \\
\hline & Opluridae & Oplurus cuvieri & LC \\
\hline Testudines & Podocnemididae & Erymnochelys madagascariensis & $\mathrm{CR}$ \\
\hline Poissons, Siluriformes & Ariidae & Arius madagascariensis & $\mathrm{LC}$ \\
\hline Gonorynchiformes & Chanidae & Chanos chanos & $L C$ \\
\hline \multirow[t]{4}{*}{ Perciformes } & Carangidae & Caranx sp. & - \\
\hline & Cichlidae & Oreochromis macrochir & - \\
\hline & & Oreochromis mossambicus & - \\
\hline & & Oreochromis niloticus & - \\
\hline
\end{tabular}

de revenus étant la culture et la pêche. Cinq familles gagnaient beaucoup plus que les autres familles enquêtées par la pratique illégale du charbonnage qui procurait un revenu irrégulier de 30000 à 360000 MGA par mois, soit entre US\$ 9,0 et US\$109,0 par mois en 2016.

La majorité des familles enquêtées (95\%) dépensaient une grande partie de leurs revenus pour l'alimentation. Les trois quarts des familles investissaient dans l'éclairage de leur maison en utilisant dans la majorité des cas des lampes à piles non rechargeables, des lampes à pétroles ou des bougies. Par ailleurs, $45 \%$ des personnes enquêtées dépensaient une partie de leurs revenus mensuels dans l'habillement et $40 \%$ constituaient une réserve pour les problèmes de santé. Seulement 35 \% des individus enquêtés dépensaient de l'argent pour l'éducation des enfants. La plupart des personnes enquêtées (90 \%) étaient conscientes de I'utilité de la forêt.

\section{DISCUSSION}

BIODIVERSITÉ ET PRESSIONS ANTHROPIQUES. La présence des

lémuriens, en particulier l'espèce en danger critique d'extinction et endémique de la région ouest de l'île Propithecus deckenii (King et Rakotonirina 2020), la présence d'oiseaux appartenant à des familles et sous-familles endémiques malgaches et de la tortue en danger critique d'extinction et endémique de la région ouest de Madagascar Erymnochelys madagascariensis (Velosoa et al. 2013) dans le site d'étude, fournissent des informations supplémentaires sur la distribution spatiale de ces animaux emblématiques de l'île. D’autres espèces devraient encore être inventoriées puisqu'à cause de l'insécurité et faute de matériel, les lémuriens nocturnes ainsi que certaines espèces d'oiseaux, les Eupleridae et les Erymnochelys madagascariensis vivants n'ont pas pu être observés.

Les données publiées associées aux Réserves spéciales de Bemarivo et Maningoza se focalisaient surtout sur la faune et la flore de Besalampy (Fishpool et Evans 2001, Schatz et al. 2001, Forthman et al. 2016). Ainsi, ce travail présente les premières informations sur les relations entre sa population locale et sa biodiversité. En effet, la plupart des familles à Besalampy vivent aux dépens de la forêt ; les zones humides associées aux aires forestières procurent à la fois l'eau pour l'agriculture et I'alimentation. Toutefois, le charbonnage à partir des plantes forestières et la culture sur brûlis, qui provoquent souvent l'érosion et la sédimentation des zones humides (Andrianandrasana et al. 2005, Styger et al. 2007), menacent la pérennité des services écosystémiques fournis par la biodiversité. Par ailleurs, la chasse des lémuriens et de la tortue Erymnochelys madagascariensis constitue une des menaces les plus importantes vis-à-vis de la biodiversité. De plus, Eulemur rufifrons subit aussi des pressions associées à la commercialisation et à la domestication.

II serait ainsi nécessaire de mettre en place d'autres activités génératrices de revenus pour subvenir aux besoins de la population locale et diminuer les pressions envers la biodiversité. Des projets de conservation durable incluant des suivies périodiques de la taille des populations de la macrofaune endémique devraient être mis en place étant donné que ces animaux sont importants pour le maintien de l'équilibre d'un écosystème, à ne citer que la dissémination de graines pour la régénération forestière par les lémuriens (Wright et al. 2011, Razafindratsima et al. 2013) ou la régulation des populations des espèces nuisibles comme les rats, par les oiseaux de proies (Thorstrom et La Marca 2000, Rasoma et Goodman 2007).

ACCORDS POUR LA CRÉATION D'UNE NOUVELLE AIRE PROTÉGÉE. La population locale vivant à Besalampy est consciente de l'utilité de la biodiversité et des effets néfastes de l'exploitation abusive de la forêt. Ainsi, les membres de la communauté locale âgés de plus de 18 ans se trouvant dans les stations d'Andranovorombahoaka, d'Antampolo, de Marofototra et d'Andranomanenty, le maire de la commune urbaine et le chef district de Besalampy en 2016 se sont entretenus avec l'association vakoka. Ils ont exprimé leur souhait de voir le développement d'un projet de conservation durable, notamment la création d'une nouvelle aire protégée (NAP) catégorie $V$ (visant à maintenir d'une manière durable l'interaction entre la biodiversité avec ses zones côtières et la population locale) à Besalampy (Dudley 2008). La NAP devrait être gérée dans un premier temps par une association ou une organisation expérimentée dans la gestion des AP qui devra former les membres de la communauté locale afin de préparer un transfert de gestion des zones tampons vers cette dernière. Un cas similaire de plan de gestion a été établi dans la NAP catégorie V du Complexe Mahavavy-Kinkony (Blackham et Avent 2018).

Les restrictions des activités de la population qui ne s'effectueront plus que dans les zones tampons ont été expliquées durant les entretiens ; et les assemblées ont signé des procès-verbaux mentionnant un avis favorable pour la création d'une NAP. D'autres études plus approfondies sur la relation entre la biodiversité et les aspects socio-économiques et culturelles de la population de Besalampy devraient être effectuées afin de mieux cadrer 
les restrictions à installer et pour bien définir les activités de compensation face aux restrictions.

\section{REMERCIEMENTS}

Nos vifs remerciements à la Mention Zoologie et Biodiversité Animale, I'Université d'Antananarivo et la Direction Générale de I'Environnement et des Forêts qui nous ont aidées dans les démarches administratives et pour nous avoir donné l'autorisation de recherche (no. 166/16/MEEF/SG/DGF/DSAP/SCB.Re) pour l'accomplissement de cette étude. Nous somme reconnaissant envers la population locale, Monsieur le Maire de la Commune Urbaine en 2016 et le Chef District de Besalampy en 2016 ainsi que son adjoint. Nos sincères gratitudes à Monsieur Botra et Bromily Lone (Dadà) qui nous ont aidés en tant qu'assistant.

\section{RÉFÉRENCES}

Albignac, R. 1970. Notes éthologiques sur quelques carnivores malgaches : le Cryptoprocta ferox (Bennett). Revue d'Ecologie (Terre et Vie) 3: 395-402 Available online $<$ https://horizon.documentation.ird.fr/exl-doc/pleins_textes/ pleins_textes_5/b_fdi_04-05/04736.pdf>

Andrianandrasana, H. T., Randriamahefasoa, J., Durbin, J., Lewis, R. E. \& Ratsimbazafy, J. H. 2005. Participatory ecological monitoring of the Alaotra wetlands in Madagascar. Biodiversity \& Conservation 14, 11: 2757-2774. $<$ https://doi.org/10.1007/s10531-005-8413-y>

Bibby, C. J., Burgess, N. D. \& Hill, D. A. 1992. Bird census techniques. Academic Press, London.

Blackham, G. V. \& Avent, T. 2018. Guide national pour la gestion durable des zones humides, Madagascar. Wildfowl and Wetland Trust, Gloucester. <https://doi.org/10.13140/RG.2.2.22782.69442>

Donati, G., Eppley, T. M., Ralison, J. M., Youssouf, J. \& Ganzhorn, J. U. 2019. Lemurs in mangroves and other flooded habitats. In: Primates in Flooded Habitats: Ecology and Conservation. K. Nowak, A. Barnett \& I. Matsuda (eds.), pp 29-32. Cambridge University Press, Cambridge.

Dudley, N. 2008. Guidelines for applying protected area management categories. IUCN, Gland. Available online <https://www.lucn.org/content/guidelinesapplying-protected-area-management-categories-0>

Fishpool, L. D. C. \& Evans, M. I. 2001. Important bird areas in Africa and associated islands: Priority sites for conservation. Pisces Publications and BirdLife International, Newbury and Cambridge. Available online <http:// datazone.birdlife.org/userfiles/file/IBAs/AfricaCntryPDFs/Madagascar.pdf>

Forthman, M., Chłond, D. \& Weirauch, C. 2016. Taxonomic monograph of the endemic millipede assassin bug fauna of Madagascar (Hemiptera: Reduviidae: Ectrichodiinae). Bulletin of the American Museum of Natural History 400: 1-152. <https://doi.org/10.1206/amnb-928-00-01.1>

Gardner, C. J. 2016. Use of mangroves by lemurs. International Journal of Primatology 37, 3: 317-322. <https://doi.org/10.1007/s10764-016-9905-1>

Goodman, S. M. et Raselimanana, A. P. 2008. Exploration et connaissance biologique des différents sites inventoriés. In: Les Forêts Sèches de Madagascar. S. M. Goodman et L. Wilmé (eds.), pp 33-45. Malagasy Nature. Available online <http://www.vahatra.mg/volume1/mn01_02.pdf>

Hawkins, C. E. \& Racey, P. A. 2008. Food habits of an endangered carnivore, Cryptoprocta ferox, in the dry deciduous forests of western Madagascar. Journal of Mammalogy 89, 1: 64-74. <https://doi.org/10.1644/06-MAMM-A-366.1>

Hawkins, F., Safford, R. \& Skerrett, A. 2015. Birds of Madagascar and the Indian Ocean Islands. Bloomsbury Publishing, London.

King, D. 1997. Reef fishes and corals: East coast of southern Africa: Seychelles, Mauritius, Comores, Madagascar and East Africa. Struik Publishers, Cape Town.

King, T. \& Rakotonirina, L. 2020. Propithecus deckenii. The IUCN Red List of Threatened Species 2020: e.T18357A115572684. <https://dx.doi.org/10.2305/IUCN.UK.2020-2.RLTS.T18357A115572684.en>
Marcus, R. R. 2001. Seeing the forest for the trees: Integrated conservation and development projects and local perceptions of conservation in Madagascar. Human Ecology 29, 4: 381-397. <https://doi.org/10.1023/A:1013189720278>

Rabenandrasana, M. 2007. Conservation biology of Sakalava rail Amaurornis olivieri an endangered Malagasy water bird and public awareness in Besalampy wetlands complex, Western Madagascar. ASITY Ligue Malgache pour la Protection des Oiseaux, Antananarivo. Available online <https:// www.africanbirdclub.org/sites/default/files/ Madagascar_Sakalava_Rail_2006_0.pdf>

Rabenandrasana, M., Zefania, S., Long, P., Seing, S. T., Virginie, M. C., et al. 2009. Distribution, habitat and status of "endangered" Sakalava rail of Madagascar. Bird Conservation International 19, 1:23-32. <https://doi.org/10.1017/S0959270908008058>

Rajemison, B. \& Goodman, S. M. 2007. The diet of Myzopoda schliemanni, a recently described Malagasy endemic, based on scat analysis. Acta Chiropterologica 9, 1:311-313. <https://doi.org/10.3161/1733-5329(2007)9[311:TDOMSA]2.0.CO;2>

Ralison, J. M. 2007. Lemur survey of the Andranomanitsy Forest, region of Besalampy, Province of Mahajanga. Lemur News 12: 36-39. Available online <https://www.dpz.eu/en/unit/library/downloads/lemur-news.html\#c3571>

Randrianarisoa, P. M., Rasamison, A. et Rakotozafy, L. 2000. Inventaire biologique dans la Réserve Spéciale de Bemarivo: Volet primatologie. Lemur News 5: 1619. Available online <https://www.dpz.eu/en/unit/library/downloads/lemur-news.htm|\#c3571>

Rasamison, A. A., Rakotozafy, L. et Raokotomanga, B. 2005. Inventaire des lémuriens dans la Réserve Spéciale de Maningoza. Lemur News 10: 20-22. Available online <https://www.dpz.eu/en/unit/library/downloads/lemur-news.htm|\#c3571>

Rasoma, J. \& Goodman, S. M. 2007. Food habits of the Barn Owl (Tyto alba) in spiny bush habitat of arid southwestern Madagascar. Journal of Arid Environments 69, 3: 537-543. <https://doi.org/10.1016/j.jaridenv.2006.10.004>

Razafimanjato, G., Sam, T. S., Rakotondratsima, M., Rene de Roland, L.-A. \& Thorstrom, R. 2013. Population status of the Madagascar Fish Eagle Haliaeetus vociferoides in 2005-2006. Bird Conservation International 24, 1: 88-99. <https://doi.org/10.1017/S0959270913000038>

Razafindratsima, O. H., Jones, T. A. \& Dunham, A. E. 2013. Patterns of movement and seed dispersal by three lemur species. American Journal of Primatology 76, 1 : 84-96. <https://doi.org/10.1002/ajp.22199>

de Rham, P. \& Nourissat, J.-C. 2004. The Endemic Cichlids of Madagascar. Association France Cichlid, Toulouse.

Schatz, G. E., Lowry II, P. \& Wolf, A.-E. 2001. Endemic families of Madagascar. VII. A synoptic revision of Leptolaena Thouars sensu stricto (Sarcolaenaceae). Adansonia 23, 2: 171-189. Available online <https://sciencepress.mnhn.fr/ sites/default/files/articles/pdf/a2001n2a1.pdf>

Soarimalala, V. 2011. Les Afrosoricides de la forêt sèche malgache. Afrotherian Conservation 8: 4-9. Available online <http://docplayer.fr/37833963Afrotherian-conservation-newsletter-of-the-iucn-ssc-afrotheria-specialistgroup.html>

Styger, E., Rakotondramasy, H. M., Pfeffer, M. J., Fernandes, E. C. M. \& Bates, D. M. 2007. Influence of slash-and-burn farming practices on fallow succession and land degradation in the rainforest region of Madagascar. Agriculture, Ecosystems \& Environment 119, 3-4: 257-269. <https://doi.org/10.1016/j.agee.2006.07.012>

Thorstrom, R. \& La Marca, G. 2000. Nesting biology and behavior of the Madagascar Harrier-Hawk (Polyboroides radiatus) in northeastern Madagascar. Journal of Raptor Research 34, 2: 120-125. Available online <https://sora.unm.edu/node/53811>

Velosoa, J., Woolaver, L., Randriamahita, Bekarany, E., Randrianarimangason, F., et al. 2013. An integrated research, management, and community conservation program for the rere (Madagascar big-headed turtle), Erymnochelys madagascariensis. In: Turtles on the brink in Madagascar: Proceedings of two workshops on the status, conservation, and biology of Malagasy tortoises and freshwater turtles. C. M. Castellano, A. G. J. Rhodin, M. Ogle, R. A. Mittermeier, H. Randriamahazo, R. Hudson \& R. E. Lewis (eds.). Chelonian Research Monographs, 6:171-177. Available online <https://chelonian.org/crm-6/> 
Vieilledent, G., Grinand, C., Rakotomalala, F. A., Ranaivosoa, R., Rakotoarijaona, J.-R et al. 2018. Combining global tree cover loss data with historical national forest cover maps to look at six decades of deforestation and forest fragmentation in Madagascar. Biological Conservation 222: 189-197. $<$ https://doi.org/10.1016/j.biocon.2018.04.008>

Wright, P. C., Tecot, S. R., Erhart, E. M., Baden, A. L., King, S. J. \& Grassi, C. 2011. Frugivory in four sympatric lemurs: Implications for the future of Madagascar's forests. American Journal of Primatology 73, 6: 585-602. <https://doi.org/10.1002/ajp.20936> 